\title{
Radiolabelled leucocytes: a new diagnostic tool in occult infection/inflammation
}

\author{
I GORDON AND G VIVIAN
}

Department of Paediatric Radiology, Hospital for Sick Children and Institute of Child Health, London

SUMmARY Autologous white blood cells were labelled with Indium $111\left({ }^{111}\right.$ In wbc) and reinjected into 18 children with suspected infection and 10 children with suspected inflammatory bowel disease aged 5 weeks to 15 years. In addition two immunosuppressed children with suspected infection received donor ${ }^{11}$ In wbc. Gamma camera images were obtained between one and four hours and 20 and 24 hours. Other appropriate investigations were also undertaken in these children. Of 18 positive ${ }^{111}$ In wbc scans 17 were true positive. There were 12 negative scans of which 9 were true negative and three were false negative. The overall results showed a sensitivity of $85 \%$, a specificity of $90 \%$, and an accuracy of $86 \%$. Used appropriately this technique forms a valuable back up investigation.

Despite improvements in diagnostic tools the occult or deep seated infection remains a challenge to clinicians. The radioactive tracer Indium $111\left({ }^{111} \mathrm{In}\right)$ labelled to a mixed population of white blood cells (wbc) has been used in adults with occult infection ${ }^{1}$ and inflammatory bowel disease. ${ }^{2}$ Since this technique does not affect chemotoxis an accurate assessment of focal wbc accumulation in pathological sites is possible. We describe the first 30 children who underwent ${ }^{111}$ In wbc scans at this hospital-in 20 children the referring physician was unable to confirm the clinical suspicion of infection by conventional methods and in 10 inflammatory bowel disease was suspected.

\section{Methods}

Following the modified method of Thakur et al., ${ }^{3}$ $10-30 \mathrm{MBq}{ }^{111} \mathrm{In}$ oxine $/ 1.73 \mathrm{~m}^{2}$ was labelled to the wbc pellet. The whole blood was reinjected into the patients. Two children received ${ }^{111}$ In labelled compatible donor wbc. All the children were scanned on a gamma camera with a medium energy collimator, and digital images were stored on a dedicated computer (Informatek Simis 3 linked to a ScintagBerthold camera). Images were obtained between one and four hours and 20 and 24 hours in all cases. Other investigations were performed as appropriate.

\section{Patients and results}

Thirty children aged between 5 weeks and 15 years underwent ${ }^{111}$ In wbc scans. These children fell into five groups.

Group A, renal. Four children underwent 6 scans. Two children in renal failure with unexplained fever had normal scans, and on clinical grounds were judged free of infection. The other two children had suspected renal carbuncles; one child had a negative scan yet had a carbuncle and the other had sequential scans because the original scan was positive (Fig. 1).

Group B, gut. Among 10 children with suspected inflammatory bowel disease 8 had concordant scans and two had false negative scans. One other child with Crohn's disease had a positive scan with an abdominal wall abscess.

Group C, bone. Seven children underwent 10 scans. Two children with multifocal osteomyelitis had sequential scans to assess the effectiveness of antibiotic treatment. In both children a changing pattern was found, enabling more appropriate treatment to be given in one and the antibiotics to be stopped in the other. Five other children had skeletal symptoms of long duration, two of these had received antibiotics for osteomyelitis. Of these five children two had normal scans (considered true negative) and three had positive scans (two true positive and one false positive).

Group D, immunocompromised. Three children with leukaemia in remission had scans; two received 
(a)

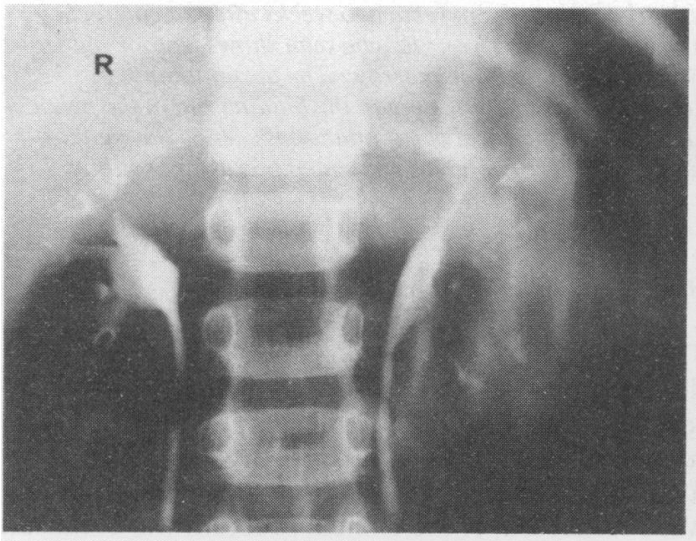

(b)
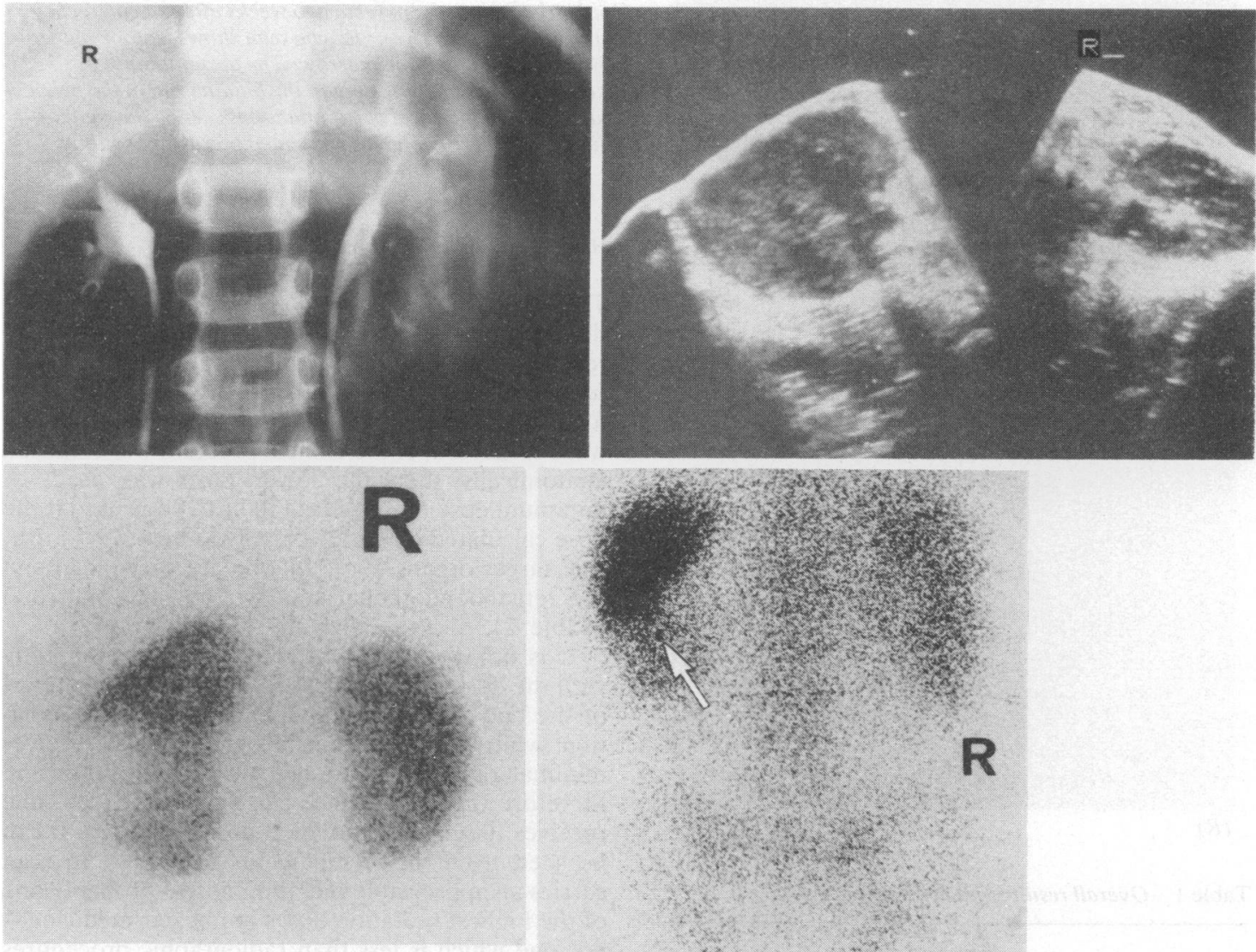

(c)

Fig. 1 Patient from Group A, renal. All the images of the right kidney are normal. (a) The IVU tomographic cut shows a large left kidney with compressed calyces; (b) the transverse prone ultrasound scan shows a swollen left kidney; (c) the posterior view of the ${ }^{99 m}$ Tc-DMSA isotope scan shows a focal deficient area on the lateral aspect of the left kidney; and (d) the ${ }^{111}$ In wbc scan, posterior view, shows isotope in the spleen, liver, and in the bone marrow of the pelvis-abnormal accumulation of isotope is noted below the spleen in the region of the left kidney (arrow).

compatible donor ${ }^{111}$ In wbc. There were two normal scans and no infection was found. One positive scan provided accurate localisation of an infection.

Group E, miscellaneous. There were three children with congenital heart disease, two of whom had a postoperative fever. There were two normal scans, considered to be true negative, and one positive scan showing a cerebral abscess. In one other child with fever after appendicectomy the scan was positive and the perirectal abscess was drained. In a fifth child with unexplained fever the scan was normal and no cause has yet been established (Fig. 2).

Pyrexias. Of the 30 children examined 12 were investigated for unexplained fever. There were 6 true positive, five true negative, and one false negative scan; no false positive scans were found.

The results of the initial scans in the 30 patients show an overall sensitivity of $85 \%$, a specificity of $90 \%$, and an accuracy of $86 \%$ (Table 1 ). 


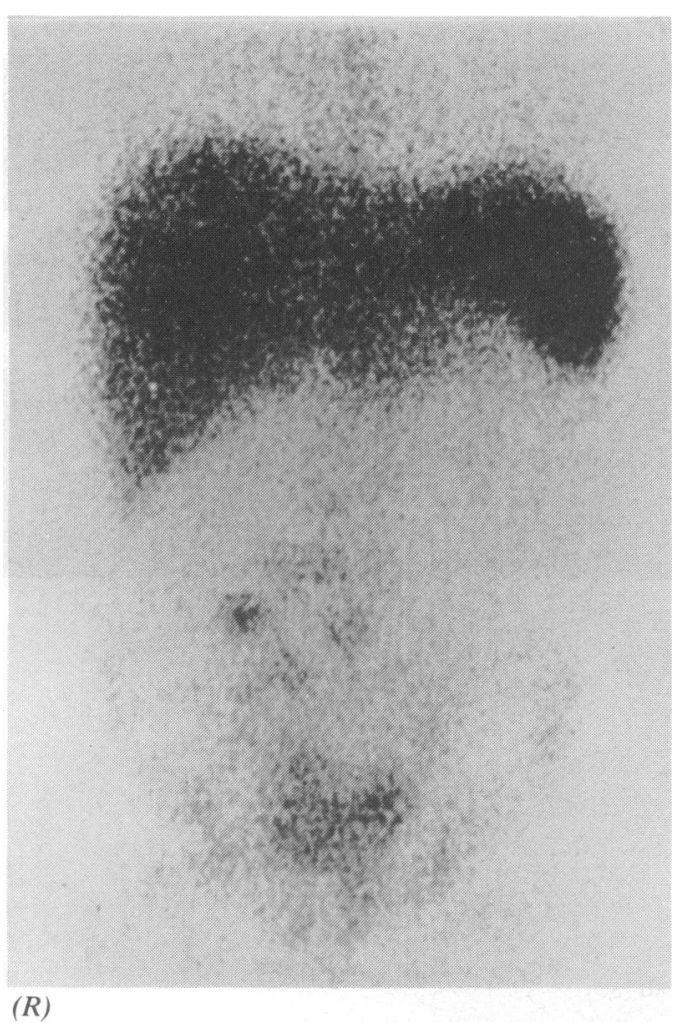

Table 1 Overall results (initial scans only)

\begin{tabular}{lccccc}
\hline & $\begin{array}{c}\text { No of } \\
\text { patients }\end{array}$ & $T P$ & $T N$ & $F P$ & $F N$ \\
\hline Group A, renal & 4 & 1 & 2 & 0 & 1 \\
Group B, gut & 11 & 8 & 1 & 0 & 2 \\
Group C, bone & 7 & 4 & 2 & 1 & 0 \\
$\begin{array}{l}\text { Group D, } \\
\text { immunosuppressed }\end{array}$ & 3 & 1 & 2 & 0 & 0 \\
$\begin{array}{l}\text { Group E, } \\
\text { miscellaneous }\end{array}$ & 5 & 3 & 2 & 0 & 0 \\
Total & 30 & 17 & 9 & 1 & 3 \\
\hline
\end{tabular}

Specificity $=90 \% ; \quad$ sensitivity $=85 \% ;$ accuracy $=86 \%$; TP=true positive $\mathrm{TN}=$ true negative, $\mathrm{FP}=$ false positive, $\mathrm{FN}=$ false negative.
Fig. 2 Patient from Group E, miscellaneous. This 15 year old girl developed a pyrexia two weeks after an emergency appendicectomy. The "In wbc scan shows isotope in the liver, spleen, and bone marrow. The accumulation of isotope over the pelvis is not in the bladder but in the pelvic cavity. A second focal accumulation is seen to the right of the spine. These two areas contained pus at laparotomy.

\section{Radiation dose}

The radiation received from a ${ }^{111}$ In wbc scan depends on the dose of ${ }^{111}$ In injected. Each child received either $10 \mathrm{mBq}(250 \mu \mathrm{Ci})$ or $30 \mathrm{MBq}(800$ $\mu \mathrm{Ci}) / 1.72 \mathrm{~m}^{2}$. The higher dose was used when no focus of infection was known or when multiple foci were suspected. If the clinical problem was localised anatomically then the lower dose was used. A minimum dose of $3.7 \mathrm{MBq}(100 \mu \mathrm{Ci})$ was used if the dose calculated on surface area was below $3.7 \mathrm{mBq}$. The target organs for ${ }^{111}$ In wbc are the spleen and the reticuloendothelial system (the bone marrow) (Table 2).

It is not possible to equate these doses directly with the dose received in radiography since that part of the body being radiographed receives the radiation while the area outside the $x$ rays receives minimal radiation. Distance is also important and therefore the skin surface closest to the $x$ ray tube receives the highest radiation dose (Table 3 ). It can be seen from this comparison that the ${ }^{11}$ In scan carries an appreciable radiation dose-probably one of the highest radiation doses in nuclear medicinebut one which is less than radiographic procedures such as long fluoroscopy or whole body computed tomography (CT).

Table 3 Radiation dosage of five minutes abdominal fluoroscopy (rads)

\begin{tabular}{lllll}
\hline & 1 year & 5 yearss & 10 years & 15 years \\
\hline Skin dose & 4 & 6 & 8.5 & 23 \\
Mean whole body & 0.2 & 0.25 & 0.3 & 0.3 \\
\hline
\end{tabular}

Table 2 Radiation dose of ${ }^{11 \prime}$ In wbc (rads)

\begin{tabular}{|c|c|c|c|c|c|c|c|c|}
\hline & \multicolumn{8}{|c|}{ Age and dosage } \\
\hline & \multicolumn{2}{|l|}{1 year } & \multicolumn{2}{|l|}{5 years } & \multicolumn{2}{|l|}{10 years } & \multicolumn{2}{|l|}{15 years } \\
\hline & $100 \mu C i^{*}$ & $250 \mu \mathrm{Cit}$ & $125 \mu C i^{*}$ & $400 \mu \mathrm{Ci} \dagger$ & $172 \mu C i^{*}$ & $550 \mu \mathrm{Cit}$ & $225 \mu C i^{*}$ & $720 \mu \mathrm{Ci} \dagger$ \\
\hline Liver & $1 \cdot 3$ & $3 \cdot 3$ & 0.9 & $3 \cdot 1$ & $0 \cdot 8$ & $2 \cdot 7$ & $0 \cdot 8$ & $2 \cdot 5$ \\
\hline Spleen & $9 \cdot 1$ & $22 \cdot 7$ & $6 \cdot 6$ & $21 \cdot 0$ & 5.9 & $18 \cdot 5$ & $5 \cdot 4$ & $17 \cdot 3$ \\
\hline Marrow & $2 \cdot 2$ & $5 \cdot 6$ & 1.6 & $5 \cdot 2$ & 1.4 & $4 \cdot 5$ & $1 \cdot 3$ & $4 \cdot 2$ \\
\hline Total body & $0 \cdot 3$ & 0.7 & 0.2 & $0 \cdot 6$ & $0 \cdot 2$ & 0.5 & 0.2 & $0 \cdot 5$ \\
\hline
\end{tabular}

* Equivalent to adult dose of $250 \mu \mathrm{Ci}$; $\dagger$ equivalent to adult dose $=800 \mu \mathrm{Ci}$. 


\section{Discussion}

Diagnosis of infection does not pose a clinical problem in most children, but the child with a complex clinical picture may tax conventional diagnostic techniques and the long list of investigations reflects the low specificity of these techniques in providing a definite answer. In this setting we use ${ }^{111}$ In wbc scans either to exclude or to diagnose an infection. This scan may also be used to assess the required duration of antibiotic treatment in certain children with a known infection.

Numerous imaging techniques have been used in adults, with varying degrees of success. ${ }^{4} \mathrm{CT}$ scans have provided useful clinical information but this is usually when a known anatomical area is pathological. In addition, the radiation dose in children is considerably higher with CT than with the ${ }^{111}$ In wbc scan, and sedation or anaesthesia is required for the younger, ill child.

Gallium 67-citrate has been used extensively to detect both infection and malignancy. Its advantage is that given as a direct IV injection, it is simpler than wbc labelling, but its disadvantage is relative non-specificity. Gallium 67-citrate has, however, proved accurate when used in the appropriate clinical setting (in paediatrics, for acute osteomyelitis). Ultrasound has proved very disappointing. Even in the children with renal carbuncles, the affected kidney simply appears enlarged with no focal abnormality on ultrasound, despite focal abnormalities on the Tc-99m DMSA and ${ }^{111}$ In wbc scans. Gas in the bowel limits ultrasound further and it has no role in bone pathology.

${ }^{111}$ In wbc labelling is relatively easy to carry out. It takes less than one hour, has been used with small volumes of blood or donor wbc, and no adverse reactions have been noted. The specificity $(90 \%)$ is dependent upon wbc chemotaxis to areas of inflammation; no attempt is made to assess this before reinjection. Trapping of the ${ }^{111}$ In wbc may normally be seen in the lungs up to 60 minutes after injection.

In one infant aged 5 weeks, ${ }^{111}$ In wbc scan proved sucessful, despite the theoretical disadvantage of potential failure of wbc migration in the newborn. It is in the neonate that the Tc-99m MDP bone scans offer little help when osteomyelitis is suspected ${ }^{5}$ and the use of ${ }^{111}$ In wbc in this group may prove important.

The ${ }^{111}$ In wbc scan offers a physiological technique to assess when to stop antibiotic treatment in those cases where the pyrexia settles rapidly and the erythrocyte sedimentation rate returns to normal. In two children with chronic multifocal osteomyelitis and one child with a renal carbuncle sequential ${ }^{111}$ In wbc scans proved an important index of active inflammation.

Group A, renal. The false negative ${ }^{111}$ In wbc scan in a child with a renal carbuncle warrants discussion. This scan was performed after two weeks of intensive antibiotic treatment when the child was asymptomatic. The distribution of ${ }^{111}$ In wbc was normal raising the possibility that two weeks treatment had been sufficient to treat his abscess adequately. A second explanation for this normal scan may be the site of the renal carbuncle-in the left upper pole-this area may well have been covered by the splenic activity on the ${ }^{111}$ In wbc scan, but this is uncertain. In a review article on the diagnostic strategy in evaluation of renal abscess Godec et al. ${ }^{6}$ state that a chronic abscess with well defined walls and without appreciable inflammatory response may give a false negative scan; unfortunately there is no supporting evidence to validate this statement. Since one third of cases of renal carbuncle are undiagnosed before surgery and mortality remains high ${ }^{7}$ the ${ }^{111}$ In wbc should be performed early in the diagnostic procedures. On ultrasound these two cases of renal carbuncle showed general enlargement of the affected kidney with no focal abnormality, an appearance not well described. It is important to emphasise the false negative scan and add some caution to negative ${ }^{111}$ In wbc scans.

Group B, gut. Full details of this group will be presented separately, since 10 children were part of a prospective study on children with suspected inflammatory bowel disease who underwent ${ }^{111}$ In wbc scan, colonoscopy, and biopsy in the same week. The 8 children who had true positive scans as well as the child with true negative scan require little discussion. Early images within four hours are essential to distinguish small from large bowel involvement. This supports the findings of Saverymuttu et al. ${ }^{8}$ who showed that in adults ${ }^{111}$ In wbc scan was a sensitive investigation for both Crohn's disease and ulcerative colitis. The two false negative results are, however, difficult to explain: both children had abnormal colonoscopy and abnormal biopsies within 48 hours of the ${ }^{111}$ In wbc scan. One child had typical active Crohn's disease on biopsy, and was in no way clinically different from the other children in the group with suspected inflammatory bowel disease. The other had a plasma cell infiltrate on biopsy with a non-specific viral colitis; this child did not show the typical features of Crohn's disease on colonoscopy or biopsy. The negative ${ }^{111}$ In wbc scan result is not surprising in view of the absence of imflammatory cells in the abnormal bowel wall on histology. 
Group C, bone. Two children with known multifocal osteomyelitis had persistent radiological or $\mathrm{Tc}^{99 \mathrm{~m}}$ MDP bone scan abnormalities, or both. In one child the return to normality of the ${ }^{111} \mathrm{In}$ wbc indicated that antibiotic treatment could be stopped, while in the other a new abnormal area developed and hyperbaric oxygen treatment was instituted. One symptomatic child with a negative ${ }^{111}$ In wbc scan had had osteomyelitis three years earlier and turned out to have a small, round cell, primary bone malignancy. One child had a false positive scan which may have been due to poor positioning on the gamma camera: this was one of the first ${ }^{11}$ In wbc scans carried out, and inexperience resulting in over reporting may also be an important factor in this case. The positive scan in a 5 week old baby with osteomyelitis is also noteworthy since it is in this age group that the usual diagnostic methods are of limited value.

Group D, immunosuppressed. All three patients had had acute leukaemia and were in remission on chemotherapy when they developed signs and symptoms resembling an infective process. Two of these children received donor ${ }^{111}$ In wbc because of severe leukopenia. Two true positive results and one true negative result in this small group is encouraging. Donor wbc have been used for ${ }^{111}$ In wbc in adults ${ }^{9}$ but there is no report of its use in children. These two children showed normal distribution of ${ }^{111}$ In suggesting that donor wbc may be useful in the leukopenic child.

Group E, miscellaneous. Three of these children were postoperative when they developed fever. In no case was an infection discovered in the thorax. It is anticipated that septic emboli to the lungs should be detected on ${ }^{111}$ In wbc scan, but further experience is needed in children with suspected subacute bacterial endocarditis before any conclusion can be drawn. When looking at the results of those children who presented with a fever, the ${ }^{111}$ In wbc scan was positive in $50 \%$ with only one false negative.

\section{Conclusion}

From these results it seems that the ${ }^{111}$ In wbc scan can be carried out in the very young (age 5 weeks) and is as effective using donor wbc as autologous wbc. The technique does not require a large volume of blood. The radiation dose is not insignificant and therefore the ${ }^{111}$ In wbc scan should form part of the backup investigations and not be used as a primary screening procedure to replace an adequate clinical examination. The specificity of $90 \%$, sensitivity of $85 \%$, and accuracy of $86 \%$ suggests that this investigation is valuable in selected problem children, but like most investigations does not achieve $100 \%$ accuracy.

The clinical indications include looking for an occult infection, judging the time to stop antibiotics in deep seated infection and in suspected inflammatory bowel disease with normal colonoscopy. The plethora of diagnostic tools available frequently tempts overuse, however, an eclectic approach to diagnostic options should be taken. When used judiciously and as a second line imaging technique it has both a high specificity and sensitivity.

Financial support from the Medical Research Council is gratefully acknowledged. We thank the clinicians of the Hospital for Sick Children and the Institute of Child Health for allowing us to study their patients and Miss M Sugarhood and Mrs G Porter for secretarial assistance.

\section{References}

${ }^{1}$ Thakur ML, Lavender JP, Amot RN. Indium"11 labelled autologous leukocytes in man. $J$ Nucl Med 1977;18:1014-21.

2 Segal AW, Ensell J, Munro JA, Serner M. Indium ${ }^{111}$ tagged leucocytes in the diagnosis of inflammatory bowel disease. Lancet 1981;ii:230-2.

${ }^{3}$ Thakur ML, Coleman RE, Welch MJ. Indium ${ }^{111}$ labelled leucocytes for the localisation of abscesses. Preparation, analysis, tissue distribution and comparison with gallium 67 citrate in dogs. J Lab Clin Med 1977;89:217-28.

4 Knöchel JQ, Koehler RR, Les TG, Welch DM. Diagnosis of abdominal abscesses with computed tomography, ultrasound and ${ }^{111}$ In leukocyte scans. Radiology 1980;138:425-31.

5 Alderson PO, Gilday DL, Wagner HN. Atlas of paediatric nuclear medicine. St Louis: CV Mosbey, 1978:24.

- Godec CJ, Tsai SH, Smith SJ, Case AS. Diagnostic strategy in evaluation of renal abscess. Urology 1981;18:535-41.

7 Thorley JD, Jones SR, Stanford JP. Perinephric abscess. Medicine 1974;53:441-9.

${ }^{8}$ Saverymuttu SH, Peters AM, Lavender JP, Hodgson HJ, Chadwick VS. Indium ${ }^{111}$ autolagous leucocytes in inflammatory bowel disease. Gut 1983;24:293-9.

9 Anstall HB, Coleman RE. Donor-leukocyte imaging in granulocytopenic patients with suspected abscesses: concise communication. J Nucl Med 1982;23:319-21.

Correspondence to Dr I Gordon, Department of Paediatric Radiology, The Hospital for Sick Children, Great Ormond Street, London WC1N 3JH.

Received 17 October 1983 